\title{
The elusive ground glass opacity, revealed
}

\author{
Melissa L. Korb, Bryan M. Burt \\ Division of Thoracic Surgery, The Michael E. DeBakey Department of Surgery, Baylor College of Medicine, Houston, TX, USA \\ Correspondence to: Bryan M. Burt, MD. One Baylor Plaza, MS:BCM 390, Baylor College of Medicine, Department of Surgery, Division of General \\ Thoracic Surgery, Houston, TX 77030, USA. Email: Bryan.Burt@bcm.edu. \\ Provenance: This is an invited Editorial commissioned by the Section Editor Dr. Jie Dai (Department of Thoracic Surgery, Shanghai Pulmonary \\ Hospital, Tongji University, Shanghai, China). \\ Comment on: Shewale JB, Nelson DB, Rice DC, et al. Natural history of ground-glass lesions among patients with previous lung cancer. Ann Thorac \\ Surg 2018;105:1671-7.
}

Submitted Aug 11, 2018. Accepted for publication Sep 10, 2018.

doi: $10.21037 /$ jtd.2018.09.41

View this article at: http://dx.doi.org/10.21037/jtd.2018.09.41

A ground glass opacity (GGO) is a radiographic finding that is defined as a hazy opacity that does not obscure underlying bronchial structures or pulmonary vessels on highresolution CT. In 2013, the Fleischner Society published a set of recommendations for the classification and management of these lesions, which was updated in 2017 $(1,2)$. GGOs may be solitary or multiple, pure ground glass or partly solid, and harbor variable malignant potential. While the majority of these radiographic abnormalities remain stable or resolve completely, a certain percentage will progress to malignancy. It is this minority group of GGOs that are of obvious concern and early identification of these lesions would facilitate clinical benefit. The balance of literature on resected GGOs has demonstrated that the majority of these lesions are pathologically benign, however a minority have malignant potential, and identification of this subset of GGOs is clinically important. In recent years, we have learned that GGOs with a solid component (partly solid GGOs) are more likely to contain invasive adenocarcinoma; whereas pure GGOs are more likely to be benign, but can also represent adenocarcinoma in situ or minimally-invasive adenocarcinoma $(3,4)$. Other studies have demonstrated additional features of GGOs that render them more susceptible to progression, including a size greater than $10 \mathrm{~mm}$, irregular or spiculated borders, and those which occur in patients with a history of cancer (5-8). For these reasons, it is important that GGOs are carefully characterized radiographically, and that a history of cancer be considered in the management of these patients. Whereas a number of recent proceedings have suggested algorithms for approaching and managing GGOs, we are still far from high-level evidence-based guidelines.

One important and often discussed question is how often and for how long to follow GGOs. Lee et al. (9) report that pure GGO lesions $\leq 10 \mathrm{~mm}$ have a volume-doubling time of more than 400 days, suggesting that yearly surveillance is sufficient. Current Fleischner Society guidelines recommend that both pure and part-solid GGOs $<6 \mathrm{~mm}$ do not require any follow up and that pure GGOs $\geq 6 \mathrm{~mm}$ should be followed every 2 years, for up to 5 years, while part-solid nodules should be surveilled annually for 5 years. Kobayashi et al. (10) showed that GGOs undergoing significant growth did so within 3 years, which suggests that all GGOs should be followed for a minimum of 3 years. The most recent iteration of the NCCN guidelines regarding follow up screening of pulmonary nodules detail recommendations for solid, partly-solid, and non-solid nodules (11). According to these guidelines, the timing of surveillance or biopsy is dictated by the size of the solid component. For a pure GGO $<20 \mathrm{~mm}$ in size, a low-dose computed tomography (LDCT) is recommended annually until the patient is no longer a candidate for definitive treatment, and for a GGO $\geq 20 \mathrm{~mm}$, a LDCT is recommended in 6 months' time.

Given the risk for progression of GGOs in patients with a history of cancer, and the elevated risk of a second lung cancer occurring in patients suffering a first lung cancer, patients with prior lung cancer are a unique population for whom GGO surveillance and treatment strategies may require special attention. In their recent manuscript, Shewale et al. (12) investigated properties of 
GGOs associated with progression or regression amongst patients previously treated for stage I-IV lung cancer. In their study population of 210 patients with a history of small cell or non-small cell lung cancer and ensuing CT evidence of pure or mixed GGOs following treatment, the authors demonstrated that $26 \%$ of these GGOs remained stable, $62 \%$ resolved, and $11 \%$ progressed. Of the $11 \%$ that progressed, 3 of 24 lesions were subsequently found to harbor malignancy. Further analysis revealed that a history of squamous cell carcinoma, small cell carcinoma, and Caucasian race were identified as predictors of GGO resolution, while a history of adenocarcinoma was the only independent predictor of GGO progression. This contribution highlights patients with previous lung cancer as a unique but relatively small population, in whom still only a minority of GGOs will progress radiographically, and in whom only a minority of these progressions will be malignant.

Once a high risk GGO is identified, the discussion of biopsy or resection is multifaceted and should include tumor location, probability of complete resection with sublobar resection, feasibility of minimally invasive resection (which should be preferred), operative candidacy and morbidity, likelihood of diagnosis with needle biopsy, and requirement of tissue diagnosis if a non-operative approach (stereotactic ablative radiotherapy) is considered. To date, the role of preoperative biopsy has not been definitively determined and has been shown to have mixed results $(5,8)$. Resection of pure (and even some part-solid GGOs) can be limited by intraoperative localization as these lesions are often not visualized or palpable, even if peripheral in location. Preoperative localization of GGOs has been shown to be useful, especially when employing thoracoscopic techniques for resection. This localization can be accomplished with placement of CT-guided hookwires or microcoils or with injection of various dyes/tracers (3). The extent of resection of pure and part-solid GGOs has been debated, although recent outcomes data have been helpful in clarifying clinical decision-making. In the past, lobectomy was thought to be indicated for all suspicious GGO lesions, with sublobar resections reserved only for high-risk patients. Recent data have shown, however, that for patients with pure GGOs or GGOs $\leq 3 \mathrm{~cm}$, sublobar resection, including segmentectomy and wedge resection, are acceptable and comparable to lobectomy for early-stage lung cancers in terms of overall survival and disease-free survival rates $(3,8,13)$. With regard to mediastinal lymphadenectomy, in clinical N0 non-small cell cancers with radiographic GGO $\leq 3 \mathrm{~cm}$, there was no difference in 5-year disease-free survival between patients who had lymph node dissection and those who did not (14). Consequently, routine lymphadenectomy for these cases was not recommended unless the nodules contained some of the high-risk features, including larger size, higher proportion of solid component, or an elevated serum CEA level (15). Despite these findings, the authors continue to recommend at least nodal sampling (if not dissection) of at least 3 nodal stations until mounting evidence accumulates in either direction.

In summary, GGOs are a diverse group of radiographic lesions for which the diagnosis of lung adenocarcinoma must be considered. The likelihood of malignancy is substantially higher in GGOs with solid radiographic components (part-solid), and, although most pure GGOs are likely to be benign, a small percentage will progress to malignancy. While we are still far from a complete understanding of these enigmatic lesions, mounting evidence, including the contribution by Shewale et al. (12) in lung cancer patients, is resolving the true nature of these radiographic lesions.

\section{Acknowledgements}

None.

\section{Footnote}

Conflicts of Interest: The authors have no conflicts of interest to declare.

\section{References}

1. Naidich DP, Bankier AA, MacMahon H, et al. Recommendations for the management of subsolid pulmonary nodules detected at CT: a statement from the Fleischner Society. Radiology 2013;266:304-17.

2. MacMahon H, Naidich DP, Goo JM, et al. Guidelines for Management of Incidental Pulmonary Nodules Detected on CT Images: From the Fleischner Society 2017. Radiology 2017;284:228-43.

3. Chen D, Dai C, Kadeer X, et al. New horizons in surgical treatment of ground-glass nodules of the lung: experience and controversies. Ther Clin Risk Manag 2018;14:203-11.

4. Qiu ZX, Cheng Y, Liu D, et al. Clinical, pathological, and radiological characteristics of solitary ground-glass opacity lung nodules on high-resolution computed tomography. Ther Clin Risk Manag 2016;12:1445-53. 
5. Lee CT. What do we know about ground-glass opacity nodules in the lung? Transl Lung Cancer Res 2015;4:656-9.

6. Kobayashi Y, Sakao Y, Deshpande GA, et al. The association between baseline clinical-radiological characteristics and growth of pulmonary nodules with ground-glass opacity. Lung Cancer 2014;83:61-6.

7. Kobayashi Y, Mitsudomi T, Sakao Y, et al. Genetic features of pulmonary adenocarcinoma presenting with groundglass nodules: the differences between nodules with and without growth. Ann Oncol 2015;26:156-61.

8. Migliore M, Fornito M, Palazzolo M, et al. Ground glass opacities management in the lung cancer screening era. Ann Transl Med 2018;6:90.

9. Lee HY, Choi YL, Lee KS, et al. Pure ground-glass opacity neoplastic lung nodules: histopathology, imaging, and management. AJR Am J Roentgenol 2014;202:W224-33.

10. Kobayashi $Y$, Fukui T, Ito $S$, et al. How long should small lung lesions of ground-glass opacity be followed? J Thorac Oncol 2013;8:309-14.

Cite this article as: Korb ML, Burt BM. The elusive ground glass opacity, revealed. J Thorac Dis 2018;10(Suppl 33):S3828S3830. doi: 10.21037/jtd.2018.09.41
11. Altorki NK, Girardi L, Skinner DB. En bloc esophagectomy improves survival for stage III esophageal cancer. J Thorac Cardiovasc Surg 1997;114:948-55; discussion 955-6.

12. Shewale JB, Nelson DB, Rice DC, et al. Natural History of Ground-Glass Lesions Among Patients With Previous Lung Cancer. Ann Thorac Surg 2018;105:1671-7.

13. Cho JH, Choi YS, Kim J, et al. Long-term outcomes of wedge resection for pulmonary ground-glass opacity nodules. Ann Thorac Surg 2015;99:218-22.

14. Moon Y, Sung SW, Namkoong M, et al. The effectiveness of mediastinal lymph node evaluation in a patient with ground glass opacity tumor. J Thorac Dis 2016;8:2617-25.

15. Zha J, Xie D, Xie H, et al. Recognition of "aggressive" behavior in "indolent" ground glass opacity and mixed density lesions. J Thorac Dis 2016;8:1460-8.

(English Language Editor: John Gray, AME Publishing Company) 